\title{
Déployer le numérique en éducation, développer les compétences
}

Federica Minichiello

\section{OpenEdition}

1 Journals

Édition électronique

URL : https://journals.openedition.org/ries/10278

DOI : 10.4000/ries. 10278

ISSN : 2261-4265

Éditeur

France Education international

\section{Édition imprimée}

Date de publication : 1 avril 2021

Pagination : 11-15

ISBN : 978-2-85420-629-6

ISSN : $1254-4590$

Référence électronique

Federica Minichiello, « Déployer le numérique en éducation, développer les compétences », Revue internationale d'éducation de Sèvres [En ligne], 86 | avril 2021, mis en ligne le 01 avril 2022, consulté le 26 mars 2023. URL : http://journals.openedition.org/ries/10278 ; DOI : https://doi.org/10.4000/ries. 10278

Ce document a été généré automatiquement le 26 mars 2023.

Tous droits réservés 


\title{
Déployer le numérique en éducation, développer les compétences
}

\author{
Federica Minichiello
}

1 La crise sanitaire a entraîné une situation inédite dans la plupart des systèmes éducatifs et a généralisé un enseignement à distance dans des conditions d'«urgence». De nombreuses études et enquêtes ont tenté d'analyser l'impact de la pandémie et d'observer les solutions entreprises pour assurer la continuité pédagogique, constatant des compétences numériques insuffisantes au sein des communautés éducatives (élèves, enseignants, familles, cadres éducatifs). Selon l'Unesco (2020), dans près de la moitié des systèmes éducatifs, y compris des pays à revenu élevé, les élèves n'avaient pas les compétences numériques requises pour suivre un enseignement à distance. $80 \%$ des pays déclaraient des compétences insuffisantes dans les familles, indépendamment des revenus des foyers. Ces chiffres montrent que, malgré des contextes politiques et socio-économiques très hétérogènes, la majorité des États se sont heurtés à des difficultés communes, tout en devant accélérer leur réflexion sur l'intégration des technologies numériques dans les pratiques éducatives et de formation tout au long de la vie.

2 En partant de quelques chiffres au niveau international, cet article propose une sélection non exhaustive d'initiatives institutionnelles et de politiques nationales illustrant différentes trajectoires et tendances pour le déploiement du numérique dans les systèmes éducatifs. Sitographie arrêtée le 29 mars 2021.

\section{Données au niveau international}

\section{International Computer and Information Literacy Study (ICILS)}

3 Cette enquête internationale a été lancée en 2013 par l'IEA (International Association for the Evaluation of Educational Achievement $)^{1}$ et en est à sa deuxième édition (2018). 
Destinée aux élèves de collège (niveau quatrième en France) elle évalue leur capacité à utiliser les technologies de l'information et de la communication et, plus précisément, leurs compétences en littératie numérique et en pensée informatique. Quelques constats ressortent des deux éditions de l'enquête: l'immersion dans un monde numérique dès la naissance ne se traduit pas automatiquement par de meilleures compétences numériques; un faible statut socio-économique est souvent corrélé à des performances inférieures dans l'utilisation du numérique; malgré des disparités liées au genre dans les métiers du numérique, les filles sont plus performantes que les garçons en littératie numérique. Les résultats de cette enquête montrent également la nécessité d'une approche holistique du numérique à l'école, avec un rôle clé joué par l'attitude des enseignants à l'égard de ces outils. Des disparités importantes existent entre les pays : si en République tchèque seuls $15 \%$ des élèves ont besoin d'un soutien important d'un adulte pour utiliser des solutions numériques, ce pourcentage s'élève jusqu'à $63 \%$ en Italie.

https://www.iea.nl/studies/iea/icils/2018

\section{TALIS 2018 (OCDE)}

4 TALIS est une enquête internationale, portée par l'Organisation de coopération et de développement économiques (OCDE), questionnant enseignants et chefs d'établissement sur leurs conditions de travail et l'environnement d'apprentissage dans leurs établissements. La dernière édition, en 2018, a mobilisé plus de 50 pays et près de 260000 enseignants. En termes de compétences numériques, l'édition 2018 montre que plus d'un jeune sur cinq, dans l'Union européenne, ne parvient pas à atteindre un niveau « de base » en compétences numériques. $40 \%$ des éducateurs se déclarent prêts à utiliser les technologies numériques dans leurs pratiques d'enseignement, avec toutefois de fortes disparités selon les pays.

http://www.oecd.org/education/talis/

\section{Citoyenneté numérique (Conseil de l'Europe)}

5 Le Conseil de l'Europe a lancé en 2016 un projet intergouvernemental sur l'éducation à la citoyenneté numérique (https://www.coe.int/fr/web/digital-citizenship-education). En 2020, l'institution a mené une enquête auprès de parents, dans 47 pays, pour analyser les préoccupations liées à la vie numérique de leurs enfants : la protection de la vie privée ressort comme l'inquiétude principale (67\% des répondants). Près d'un parent sur deux exprime un besoin d'accompagnement pour choisir les plateformes éducatives les plus appropriées pour ses enfants, et certains indiquent le souhait d'un système de classification comme celui pour les jeux en ligne (PEGI).

https://bit.ly/3qGlUss

\section{Actions au niveau international}

\section{Coalition mondiale pour l'éducation}

6 La crise sanitaire a exacerbé une fracture numérique mondiale déjà existante ${ }^{2}$, avec près de la moitié de la population mondiale éloignée d'un accès internet (pour 
différentes raisons : obstacles socioculturels, barrière économique et/ou technologique, manque de compétences ou de formation, etc.), dans un contexte de pénurie mondiale d'enseignants bien formés et qualifiés. Lancée en 2020, la Coalition mondiale pour l'éducation est un partenariat mondial d'ampleur inédite réunissant 140 membres du système onusien, de la société civile, du monde académique et du secteur privé. Son objectif est de répondre à la situation de crise par une stratégie en trois axes: l'amélioration de la connectivité, la formation des enseignants, l'attention pour les publics à besoins spécifiques. Tous les membres de la coalition s'engagent à proposer des outils et des services, gratuitement, pour assurer la continuité pédagogique.

https://bit.ly/20Xnuc4

\section{Plan d'action européen en matière d'éducation numérique}

7 Après une phase de consultation publique, la Commission européenne a publié son plan d'action «numérique » pour la période 2021-2027. Ce programme est structuré autour de deux priorités stratégiques: favoriser le développement d'un écosystème performant (infrastructure, équipement, renforcement des capacités, contenus de qualité, plateformes sécurisées, etc.) et renforcer les aptitudes et compétences numériques pertinentes pour la transformation numérique. Parmi les actions proposées, on peut évoquer une étude de faisabilité pour la création d'une plateforme européenne de partage des ressources éducatives en ligne «labelisées », l'appui par le programme Erasmus+ de plans de transformation numérique, le renforcement des capacités des enseignants dans l'utilisation du numérique grâce à des outils comme la plateforme Teacher Academy d'Erasmus (https://www.teacheracademy.eu/) et l'outil d'auto-diagnostic SELFIE: Self-reflection on Effective Learning by Fostering the Use of Innovative Educational Technologies ( https://ec.europa.eu/education/schools-godigital_fr).

https://bit.ly/3v3ihjI

\section{Organisation internationale de la Francophonie}

8 L'Organisation internationale de la Francophonie publie, tous les deux ans, un rapport sur la francophonie numérique avec des exemples de bonnes pratiques dont les pays francophones peuvent s'inspirer. Début 2020 l'organisation a signé un accord-cadre avec Smart Africa (https://smartafrica.org/), initiative réunissant environ 30 pays africains, vouée à l'accélération du développement socio-économique du continent grâce aux TIC. Les thèmes de coopération entre les deux institutions sont les suivants : le développement de la connectivité et de l'accès aux infrastructures numériques, en privilégiant le développement d'une connectivité intra-africaine, la formation au numérique et la promotion des contenus numériques en langue française, la promotion de l'économie digitale.

https://www.francophonie.org/

\section{IMS Global Learning Consortium}

IMS est une ONG à but non lucratif composée d'environ 150 membres, acteurs de l'innovation numérique, réunis autour de l'objectif de développer l'utilisation des technologies au service de l'apprentissage et de l'éducation. L'association œuvre en 
particulier pour promouvoir l'interopérabilité des systèmes utilisés et le développement de normes et de référentiels permettant de décrire les technologies éducatives. Elle promeut une architecture et un écosystème plug-and-play ${ }^{3}$, sur lesquels des produits innovants peuvent être déployés et, surtout, fonctionner ensemble, sans difficultés d'interconnexion.

http://www.imsglobal.org/

\section{Exemples au niveau national}

\section{France}

La France a mené une consultation nationale qui a conduit aux États généraux du numérique en novembre 2020. Résultat de cette concertation, quarante actions ont été priorisées et mises au cœur de la stratégie numérique nationale, comme la conception d'espaces numériques personnels pour l'éducation et l'orientation (portfolio de compétences), un " pass connexion éducation " pour démocratiser l'accès aux outils et aux ressources éducatives en ligne, le renforcement du cadre de confiance à travers un code de conduite pour les entreprises de la filière, la mise en œuvre de «territoires numériques éducatifs ${ }^{4}$ » pour former au et par le numérique, l'accompagnement d'opérations innovantes comme des «challenges» dans le domaine de l'intelligence artificielle, etc.

https://bit.ly/30FbFu1

\section{Estonie}

11 Dès 2014, l'Estonie a déployé une stratégie nationale et multisectorielle de transformation numérique de sa société. En éducation, l'accent a été mis sur le développement de compétences numériques, en intégrant des modules ad hoc dans la formation des enseignants et dans les programmes scolaires. En 2019, selon Eurostat, $96 \%$ des 16 à 19 ans disposaient des compétences numériques « de base » et l'intégralité des établissements scolaires bénéficiaient d'une infrastructure numérique, principalement dans le nuage, avec un choix d'outils proposés aux enseignants et aux élèves. Plusieurs projets pilotes ont été lancés, récemment, pour développer des applications d'intelligence artificielle en éducation, visant à construire des modèles prédictifs pour l'évaluation diagnostique et à personnaliser les ressources d'apprentissage.

https://e-estonia.com/education-nation/

\section{Danemark}

12 Le Danemark ressort de l'enquête TALIS comme le pays au plus grand nombre d'enseignants laissant les élèves utiliser les TIC pour des projets en classe (58 \% dans le $1^{\text {er }}$ degré, $90 \%$ au collège) et utilisant des outils numériques dans leurs pratiques quotidiennes d'enseignement. Cette démocratisation des usages est accompagnée d'un taux d'équipement très important, d'une politique nationale promouvant le système dit «AVEC » (Apportez votre équipement personnel de communication) et de l'utilisation d'ordinateurs pour une grande partie des cours, dès l'école primaire. Parmi les 
pratiques intéressantes, on peut citer l'enseignement de la "compréhension technologique ", discipline à la croisée des sciences humaines, des disciplines créatives et informatiques, qui souhaite doter les élèves d'une bonne compréhension des pratiques liées au numérique et d'un regard critique sur l'impact de la technologie.

https://bit.ly/3lhHG4U

\section{Kenya}

Selon la Banque mondiale, le secteur des TIC au Kenya a connu une croissance annuelle moyenne de 10,8\%, sur les cinq dernières années. Le pays accueille d'ailleurs le centre d'excellence AICE pour le numérique et l'intelligence artificielle (https:// aiceafrica.com/), qui vise à promouvoir la recherche et développement, l'expertise et l'utilisation de l'intelligence artificielle sur le continent africain. Dans le cadre de sa stratégie nationale à l'horizon 2030, le Kenya s'est doté du programme Digital Literacy Programme (DLP) porté par le ministère de l'information, des communications et des technologies. La première phase du programme a permis de toucher plus de 20000 écoles primaires, de distribuer plus d'un million d'appareils avec du contenu numérique "labellisé » préinstallé et de former plus de 200000 enseignants aux pratiques numériques. La deuxième phase (2019-2023) vise à élargir ces actions aux niveaux de classe successifs, et à l'enseignement spécialisé.

http://icta.go.ke/digischool/

\section{Uruguay}

14 En 200,7 l'Uruguay a lancé le programme CEIBAL, afin de fournir à tous les élèves et enseignants des écoles primaires publiques un accès gratuit à un ordinateur portable. Ce plan d'action a été accompagné de la mise à disposition de points de connexion internet dans les places publiques du pays, la création d'une plateforme centralisée pour les manuels scolaires numériques, la formation continue des enseignants. Cet investissement soutenu dans le numérique, depuis dix ans, a permis une résilience accrue du pays face à la pandémie et une meilleure continuité pédagogique, par rapport à des pays voisins. Toutefois, comme d'autres expériences d'«un ordinateur par enfant ", les résultats du programme semblent contrastés : malgré une augmentation significative de l'accès aux ordinateurs, et une réduction de la fracture numérique, l'écart de scolarisation entre les élèves des écoles privées et publiques semble persister. Parmi les élèves de l'enseignement secondaire, ceux ayant été exposés au programme depuis leur enfance ne semblent pas être plus enclins à choisir une orientation dans le domaine scientifique ou technologique.

https://www.ceibal.edu.uy/es 


\section{NOTES}

1. L'IEA, Association internationale pour l'évaluation du rendement scolaire, est une organisation indépendante, à but non lucratif, qui mobilise un réseau de plus de 60 pays et de 100 systèmes éducatifs. L'association est notamment à l'origine des enquêtes internationales sur les mathématiques et les sciences (TIMSS), la lecture (PIRLS), etc.

2. Voir notre article dans la Revue internationale d'éducation de Sèvres $n^{\circ} 84$ (2020) : "Un accès internet pour tous : enjeux, solutions ». https://journals.openedition.org/ries/9703

3. L'expression "plug and play» (brancher et utiliser) désigne un système qui sait reconnaître des périphériques ou des logiciels sans nécessité de redémarrer un ordinateur.

4. Bénéficiaire du Programme d'investissements d'avenir, ce programme est porté par Réseau Canopé, en partenariat avec le Secrétariat général pour l'investissement, la Caisse des dépôts et des consignations, la Trousse à projets et les collectivités locales: https:// www.education.gouv.fr/les-territoires-numeriques-educatifs-306176

INDEX

Palabras claves : competencias digitales, ciudadanía, digital

Keywords : digital skills, citizenship, digital

Mots-clés : compétences numériques, citoyenneté, numérique

Index géographique : Pays de l'OCDE, Union européenne, France, Estonie, Danemark, Kenya, Uruguay

\section{AUTEUR}

\section{FEDERICA MINICHIELLO}

Federica Minichiello est directrice par intérim du centre de ressources et d'ingénierie documentaires et responsable du laboratoire numérique de l'éducation à France Éducation International. Courriel : minichiello@france-education-international.fr 